DOI: https://doi.org/10.46296/rc.v2i3.0034

\title{
Big data y marketing de experiencias Big data and experience marketing
}

\author{
Zanella-Martínez Leonardo Mauricio \\ Investigador ecuatoriano independiente. Profesor universitario de \\ instituciones de Educación Superior. Consultor empresarial. Ecuador. \\ Correo: leozanella80@gmail.com \\ ORCID ID: https://orcid.org/0000-0002-2388-5767 \\ Chiriboga-Mendoza Fidel Ricardo \\ Profesor Titular de la Universidad Laica Eloy \\ Alfaro de Manabí. Ecuador, ULEAM. Manta, Ecuador. \\ Correo: fidel.chiriboga@uleam.edu.ec \\ ORCID ID: https://orcid.org/0000-0002-3378-8610
}

\author{
Zambrano-Pilay Enrique Cristóbal \\ Universidad Laica Eloy Alfaro de Manabí. Ecuador, ULEAM. Manta, Ecuador. \\ Correo: eczp_1997@hotmail.com \\ ORCID ID: https://orcid.org/0000-0002-9329-0929
}

\begin{abstract}
RESUMEN
Hoy por hoy, con el auge del internet y de la era digital, las empresas tienen el deber de adaptar sus estrategias y sistemas metodológicos de comercio para lograr crear y mantener la competitividad en los mercados existentes. En este marco contextual, el marketing de experiencias es una de las formas del marketing contemporáneo que busca crear dependencia a través de determinado producto o servicio en los consumidores, ofreciendo recursos empíricos para crear preferencias en los consumidores de una marca con respecto a otra marca. El marketing de experiencias, siendo una de las tantas formas existentes del marketing relacional, ha sido modificado por las empresas para ser fácilmente transmitido a los consumidores a través de estrategias de comunicación digital en entornos digitales, como en el caso de los sitios web, redes sociales etc. Este proceso de recuperar, clasificar y detallar la información recopilada se conoce como "análisis de big data" y consiste en la adaptación de un conglomerado de información que tiene una estructura de datos integrada compleja con las notorias dificultades para buscar, almacenar, analizar, proteger, transferir y visualizar los datos recopilados. En particular, el análisis de big data es muy importante para las empresas con la base del comercio electrónico y el comercio digital, que pueden estudiar el comportamiento del consumidor, luego realizar un análisis detallado y finalmente obtener importantes beneficios en la competencia en un mercado determinado.
\end{abstract}

Palabras claves: Big data, marketing, redes sociales, empresa, consumidor. 


\section{ABSTRACT}

Today, with the rise of the internet and the digital age, companies have the duty to adapt their strategies and methodological trading systems to create and maintain competitiveness in existing markets. In this contextual framework, experience marketing is one of the forms of contemporary marketing that seeks to create dependence through a certain product or service in consumers, offering empirical resources to create preferences in consumers of a brand with respect to another brand. Experience marketing, being one of the many existing forms of relationship marketing, has been modified by companies to be easily transmitted to consumers through digital communication strategies in digital environments, as in the case of websites, networks social etc. This process of retrieving, classifying and detailing the information collected is known as "big data analysis" and consists of the adaptation of a conglomerate of information that has a complex integrated data structure with the notorious difficulties to search, store, analyze, protect, transfer and view the collected data. In particular, big data analytics is very important for companies with e-commerce and digital commerce foundation, which can study consumer behavior, then perform detailed analysis and finally obtain significant benefits in competition in a given market.

Keywords: Big data, marketing, social media, business, consumer.

\section{INTRODUCCIÓN}

Hoy en día, la cantidad de información recopilada de múltiples tecnologías de gestión de datos se ha convertido en una tendencia en el ecosistema de marketing digital y ha crecido a un ritmo sin precedentes en los últimos años. Derivados como el análisis de big data son críticos para las empresas con base en el comercio electrónico y digital, estas empresas deben estudiar el comportamiento del consumidor, seguido de un análisis detallado de posibles cambios en sus intenciones de compra en mercados específicos basados en el comercio electrónico, donde sea posible. para obtener cantidades significativas de datos a través de transacciones en línea u otros recursos. Las empresas de comercio electrónico a menudo se asocian con datos estructurados y no estructurados. En ese sentido, el marketing relacional o marketing de experiencias es un proceso más complejo de lo que sugiere su definición central. Desde un punto de vista filosófico, ha pasado de un simple enfoque en la comprensión de las necesidades de los clientes a una exploración intrínseca de los estilos de vida de los clientes, incluyendo sus valores, trabajos por hacer, estados de necesidad y requerimientos. En un nivel práctico, la multiplicidad de 
datos digitales en tiempo real sobre los clientes ha allanado nuevas formas para comprender sus comportamientos. En conjunto, la complejidad de la segmentación de clientes que se manifiesta hoy en día, lo ha convertido en un tema delicado para los especialistas en marketing. La segmentación del mercado debe abordar las realidades de los clientes actuales con conocimientos digitales y alinear las funciones comerciales con los segmentos de clientes en los que se puede enfocar. Centrarse en el cliente es la palabra operativa y las organizaciones son más efectivas con estas estrategias; puesto que, de esta forma, las organizaciones son más eficaces para satisfacer los requisitos actuales de ventas y participación en el mercado y los requisitos a largo plazo de expansión y crecimiento empresarial. El papel del Big-Data en el análisis de los requisitos de los clientes y luego en la segmentación se está volviendo imprescindible.

\section{PERSPECTIVAS DEL BIG DATA Y EL MARKETING DE EXPERIENCIAS}

En primera instancia, cabe destacar que la cantidad de información recopilada a partir de múltiples técnicas de administración de datos es una tendencia creciente que crece constantemente a un ritmo sin precedentes. Consiste en la adaptación de un conglomerado de información que tiene una estructura de datos integrada compleja con las notorias dificultades para buscar, almacenar, analizar, proteger, transferir y visualizar los datos recopilados. Este proceso de recuperar, clasificar y detallar la información recopilada se conoce como "análisis de big data". En este contexto, la analítica de Big Data es de vital importancia en las empresas con bases de comercio electrónico y digital para estudiar el comportamiento del consumidor, posteriormente realizar análisis detallados, y en última instancia obtener importantes beneficios con respecto a la competencia en un mercado determinado (Sayyad Et al, 2019). Múltiples herramientas producen una gran cantidad de información, como en el caso específico del comercio electrónico, así como transacciones digitales, redes sociales, call center y en última instancia dominios de telecomunicaciones. 
Particularmente, en los últimos años la producción e intercambio de datos se ha presentado como una tendencia creciente, afectando significativamente el ecosistema del comercio electrónico. Por su naturaleza, el comercio electrónico se concibe como uno de los principales medios en los que se desarrolla de manera más eficiente la analítica de big data, al tiempo que demuestra su viabilidad para satisfacer las diferentes necesidades de los mercados contemporáneos, así como diversos temas similares como la variabilidad en el comportamiento del consumidor, múltiples desafíos de retención de clientes y, en última instancia, mayores ganancias. La información recopilada de los consumidores incluye datos como nombre, edad, dirección, sexo, etc. Mientras que, por su parte, los datos no estructurados incluyen información generada como resultado de las preferencias del consumidor, como por ejemplo, Me gusta y enlaces recientes en Facebook. así como tweets en el caso de Twitter, etc. Actualmente, una gran parte de la población mundial se dedica principalmente a adquirir y promover bienes, servicios y productos a través de plataformas de comercio por Internet. En este sentido, las empresas que desarrollan su actividad comercial en base a los beneficios del comercio electrónico han desarrollado un ecosistema completo para adquirir y vender productos a través de la modalidad de comercio online. Para optimizar el proceso de análisis del comportamiento del consumidor, cada transacción realizada por un determinado cliente se almacena, recopila y analiza en detalle. Por tanto, es necesario afirmar que el "Big data" es fundamentalmente útil en las empresas de comercio electrónico.

Bajo esta premisa, Lies, Jan (2019) denota la gran cantidad y disponibilidad de aplicaciones digitales, que a su vez configuran el paradigma actual del marketing digital y big data, adoptando el término "inteligencia de marketing" desde el punto de vista de la técnica de marketing. como resultado de lo cual el autor describe la inteligencia de marketing como una parte esencial de las técnicas de ingeniería social.

\section{EL BIG DATA Y EL MARKETING DE EXPERIENCIAS EN EL CONTEXTO GLOBAL}

La disponibilidad de múltiples aplicaciones móviles y las interdependencias de estas técnicas socio-digitales muestran que el big data y 
la inteligencia de marketing se han consolidado como sucesores del marketing clásico en el ecosistema del e-marketing. Naturalmente, como resultado de esta tendencia, el marketing contemporáneo está experimentando un cambio sustancial en su estigma metodológico, cultural y técnico que aumenta y amplifica el marketing de salida convencional con el marketing de entrada moderno.

El principal objetivo del big data en el ecosistema del marketing digital es procesar la información en tiempo real de una forma más rápida y precisa. En particular, los sistemas de análisis y difusión de datos deben cumplir altos estándares en cuanto a la velocidad que respetan, tanto en el procesamiento de la información recopilada como en el análisis de datos, para garantizar evaluaciones más ágiles. En este contexto, el análisis de datos registra información específica del consumidor, como la frecuencia de uso de tarjetas de crédito y compras digitales.

En este contexto, Lukosius y Hyman (2019). argumentan que el big data es más compatible con un enfoque teórico y empírico que con un enfoque teórico aislado para el desarrollo de la teoría del marketing. Nuestra exposición procede de la siguiente manera. Primero, presentamos ambos enfoques y big data.

En ese sentido, los autores discuten tres fuentes establecidas de la teoría del marketing contemporáneo, y en las que el big data se posiciona como una cuarta fuente. Específicamente, en los últimos años, el llamado enfoque de teoría en silos se ha mantenido como el estándar para el ecosistema de marketing contemporáneo. Sin embargo, la adopción de este enfoque ha hecho imposible que los especialistas en marketing adopten y adapten varias alternativas viables, como en el caso específico del enfoque teórico y empírico, que sugiere teorías basadas en las particularidades empíricas observadas en el ecosistema. de marketing.

A pesar de ello, la recogida de información se está produciendo cada vez de forma más generalizada gracias al potente hardware de las nuevas supercomputadoras y los sofisticados y complejos algoritmos desarrollados en base a software de programación están impulsando la nueva era del Big Data, que son datos complejos y masivos. conjuntos, pero que se recopilan de manera 
eficiente de múltiples fuentes, mucho más viable para el enfoque empírico. En este sentido, las teorías del marketing surgieron a partir de la experiencia de gestión y la actividad investigadora en el marketing y disciplinas afines, como, por ejemplo, en el contexto de la economía, donde el big data representa una fuente complementaria de información.

\section{EL BIG DATA Y EL MARKETING DE EXPERIENCIAS EN EL CONTEXTO LATINOAMERICANO}

A nivel de Latinoamérica la investigación de big data, así como su impacto en las industrias, constituyen sin duda uno de las áreas de estudio más importantes en el ecosistema del e-marketing de la última década. A pesar de múltiples investigaciones sobre la aplicación del análisis de datos, como en el caso específico de la minería de datos, las estadísticas y los sistemas de recolección de información, las investigaciones anteriores no se enfocaron en el potencial del Big Data en el contexto del marketing digital. No fue hasta la última década que las industrias comenzaron a prestar más atención al desarrollo de big data.

Como se ha evidenciado en múltiples investigaciones, en la última década, la cantidad de datos ha aumentado exponencialmente en conjunto con el desarrollo de nuevas tecnologías para la generación, producción, clasificación, almacenamiento y análisis de datos, particularmente en el caso específico de los dispositivos inteligentes que funcionen sobre la base de una nube de datos utilizando Internet y nuevas tecnologías de bases de datos, como Google Drive. Estas herramientas de almacenamiento de datos y dispositivos basados en Internet, como teléfonos inteligentes, tabletas y dispositivos de uso medido, se utilizan comúnmente en todo el mundo tanto por los consumidores como por todo tipo de industrias para comercializar sus productos o servicios. Como predijo International Data Corporation, se estima que para el 2025, habrá aproximadamente 175 Zettabytes de datos aumentados de los 41 Zettabytes en 2019 (Wang, W. Y. C., \& Wang, Y, 2020).

Por este motivo, la investigación más reciente derivada del contexto del big data en el ecosistema del marketing digital requiere de un equipo de 
investigación multidisciplinar, expertos del sector con competencias y conocimientos informáticos profesionales. Además, los macrodatos son propensos a sesgos, y los expertos multidisciplinarios pueden aliviar en gran medida este sesgo. Por lo tanto, los académicos contemporáneos que estén considerando confiar en herramientas de análisis relacionadas con big data (como minería de datos, computación cognitiva, redes neuronales e inteligencia artificial) para estudiar teorías contemporáneas y métodos empíricos deben adquirir habilidades más allá de la formación empresarial tradicional.

\section{CONCLUSIONES}

La integración de la tecnología en las estrategias comerciales aumentará la complejidad de las comunicaciones de marketing, por lo que se necesita con urgencia un análisis avanzado del desempeño del marketing. El rápido desarrollo de los métodos de atribución de marketing ha creado lagunas en la descripción sistemática y la interpretación funcional de los métodos.

En particular, el análisis de big data es fundamental para las empresas con una base para el comercio electrónico y el comercio digital, que deben estudiar el comportamiento del consumidor, luego realizar un análisis detallado $\mathrm{y}$, en última instancia, obtener importantes beneficios competitivos en un mercado específico.

\section{REFERENCIAS}

A.D. Nazarov (2019). Big Data Driven Marketing. Proceedings of the International Scientific and Practical Conference on Digital Economy (ISCDE) on November 07, 2019.

Dimitrios Buhalis, Katerina Volchek (2021). Bridging marketing theory and big data analytics: The taxonomy of marketing attribution. International Journal of Information Management.

Dytman-Stasieńko, Agnieszka \& Węglińska, Agnieszka. (2018). Big Data, Digital Marketing and Trendwatching. 10.23817/2018.bdata. 
Erevelles, S., Fukawa, N., \& Swayne, L. (2016). Big Data consumer analytics and the transformation of marketing. Journal of Business Research, 69(2), 897-904. doi:10.1016/j.jbusres.2015.07.001.

Kemelor, P. (2015). Digital Data Grows into Big Data. IT Professional, 17(4), 4248. doi:10.1109/mitp.2015.69.

Konks, V. \& Krasnov, E. \& Sumarokova, E. (2019). Digital marketing and sales technologies: big data and synergy effect. Vestnik Universiteta. 46-54. 10.26425/1816-4277-2019-8-46-54.

Lies, Jan (2019). Marketing Intelligence and Big Data: Digital Marketing Techniques on their Way to Becoming Social Engineering Techniques in Marketing. International Journal of Interactive Multimedia and Artificial Intelligence in June, 2019.

Sayyad, S., Mohammed, A., Shaga, V., Kumar, A., \& Vengatesan, K. (2019). Digital Marketing Framework Strategies Through Big Data. Proceeding of the International Conference on Computer Networks, Big Data and IoT (ICCBI - 2018), 1065-1073. doi:10.1007/978-3-030-24643-3_127.

Vaidas Lukosius, Michael R. Hyman (2019). Marketing Theory And Big Data. The Journal of Developing Areas, Volume 53, Number 4, Fall 2019.

Wang, W. Y. C., \& Wang, Y. (2020). Analytics in the era of big data: The digital transformations and value creation in industrial marketing. Industrial Marketing Management. 